EPJ Web of Conferences 61,04001 (2013)

DOI: $10.1051 /$ epjconf/ 20136104001

(C) Owned by the authors, published by EDP Sciences, 2013

\title{
Multi-waveband Behavior of Blazars
}

\author{
Alan P. Marscher ${ }^{1}$ \\ ${ }^{1}$ Institute for Astrophysical Research, Boston University, 725 Commonwealth Ave., Boston, MA 02215 USA e-mail: marscher@ \\ bu.edu
}

\begin{abstract}
The author reviews recent progress toward understanding blazars that multi-waveband monitoring observations have advanced. The primary techniques include the compilation of multi-waveband light curves, multi-epoch VLBI images at radio wavelengths, plots of linear polarization vs. time at radio through optical wavelengths, and spectral energy distributions (SEDs). Correlations and the coincidence or lag of events across wavebands and in the images indicate where the events take place relative to the "core" that lies $\gtrsim 0.5 \mathrm{pc}$ from the central engine. Rotations of the polarization electric vector suggest a helical geometry of the magnetic field upstream of the millimeter-wave core, while rapid fluctuations in degree and position angle of polarization imply that the jet plasma is turbulent in and downstream of the core. The author is developing a numerical model that simulates the emission from such turbulence as it interacts with a conical standing shock in the core region.
\end{abstract}

\section{Introduction}

Blazars are well known for their extreme properties: they are the most luminous objects in the universe that last longer than a few minutes, radiate across nearly the entire electromagnetic spectrum, often have much higher luminosities at $\gamma$-ray than at lower frequencies, exhibit motions that appear to be faster than light, and change their flux and polarization on quite short time-scales. How they manage to perform for astronomers in such a fashion has been one of the greatest cosmic mysteries, the solution to which has proved to be extremely difficult to unravel because of the complex array of phenomena involved. At this stage, we have determined that the essence of blazars lies in their high-energy, magnetized plasmas that flow at near-light velocities in the highly collimated structures we call "jets." Although we have some basic understanding of some aspects of jets, it is safe to say that we never would have predicted their existence from first principles. Indeed, it has been challenging to understand how a central engine that is basically a monstrous black hole attracting and accreting surrounding gas produces such ultra-fast, collimated outflows.

Multi-waveband observations play a central role in the struggle to figure out the physical properties of jets and how the well-collimated relativistic flows are generated. The great hope is to use details of variations in flux, polarization, structure, and spectral energy distributions (SEDs) to probe the physics of jets as close to the central engine as possible. The questions that such programs seek to answer include:

1. Is there strong evidence for most theorists' belief that jets are launched, accelerated, and collimated by the twisting of magnetic field lines owing to the differential rotation of plasma near the black hole?

2. How are the particles that produce the high-energy emission accelerated - at shock fronts, via turbulence, or during magnetic reconnections?

3. What are the dynamics of the flow in the jet? How common are moving and stationary shocks? What role do instabilities play in the emission and structure of jets?

4. What is the jet power? This is important for knowing what fraction of the jet's energy is deposited into the galaxy and its surroundings, possibly causing feedback that regulates galaxy growth.

5. Are the $\gamma$ rays from inverse Compton scattering by relativistic electrons, secondary processes initiated by protons, or even proton synchrotron radiation? If protons are responsible for much of the luminosity, the power requirements are considerably higher than for radiation by electrons.

6. Do outbursts at $\gamma$-ray and lower frequencies occur mostly on parsec scales? If so, how can the $\gamma$-ray flux be so highly variable when it arises in a region so distant from the black hole? If only a small fraction of the jet cross-section is involved, how can such a small region generate such a high apparent luminosity?

This review highlights some of the recent progress in our physical description of jets in blazars enabled by 
multi-waveband observations. Emphasis is given to studies and results that are not reported by others at the conference; the reader is referred to the corresponding papers in these proceedings. The author apologizes to the authors of many enlightening studies that are not mentioned here because of space limitations.

\section{Multi-waveband Light Curves}

In the past, blazar monitoring programs often had bad reputations among telescope time allocation committees for using a lot of telescope time while only publishing the results after years of data accumulation. Rather than solving the outstanding problems, these programs usually generated new questions. The basic reason for this is the complexity of the physical processes in jets and the incomplete time and frequency coverage of past observations. The latter has been overcome to a great extent by the availability of the Fermi Gamma-ray Space Telescope and its Large Area Telescope (LAT).

Fermi is usually in scanning mode, surveying the entire sky every three hours. There is a very small sun avoidance zone, so the light curves that it generates are essentially continuous year-round, with time resolution that can be intra-day when a blazar undergoes a $\gamma$-ray outburst exceeding several times $10^{-6}$ phot $\mathrm{cm}^{-2} \mathrm{~s}^{-1}$. The effective frequency range is about two orders of magnitude, even greater when the flux is extremely high, so the slope of the $\gamma$-ray SED can be measured to reasonable accuracy. The result is that now, just over five years since Fermi began science operations, we have an excellent characterization of the $\gamma$-ray behavior of tens of blazars and at least mean flux levels and parameters of the time-averaged $\gamma$-ray SED of hundreds more.

It is instructive to browse through the $\gamma$-ray light curves, based on an unrefined analysis of Fermi LAT data, at webpage http://fermi.gsfc.nasa.gov/ssc/data/access/ lat/msl_lc/. Blazars featured at this site were selected based on strong detection by EGRET on the Compton Gamma Ray Observatory or a LAT-measured flux that exceeded $1 \times 10^{-6}$ phot $\mathrm{cm}^{-2} \mathrm{~s}^{-1}$ at some time. A small number of objects that do not meet these criteria but were anticipated to be interesting are included as well. The light curves on the right of the webpage, with one-week binning of the data, are quite striking. Immediately apparent to the eye is the fact that none of the blazars has a roughly constant $\gamma$-ray flux. There are some quasars and BL Lac objects whose $\gamma$-ray flux never sinks below the detectable (with one-week integration) level of $\sim 2 \times 10^{-7}$ phot $\mathrm{cm}^{-2} \mathrm{~s}^{-1}$, but those fluctuate between strong and weak. This volatility of the $\gamma$-ray emission is quite similar to that seen at lower frequencies in blazars. We can then ask the question of whether there is a strong relationship between the $\gamma$-ray and lower-frequency variations in the hope that this will lead to insights into the physical conditions in the relativistic jet.

Usually when we analyze the synchrotron emission at optical frequencies, including relating the time-scale of the variations in flux to the time-scale for radiative energy

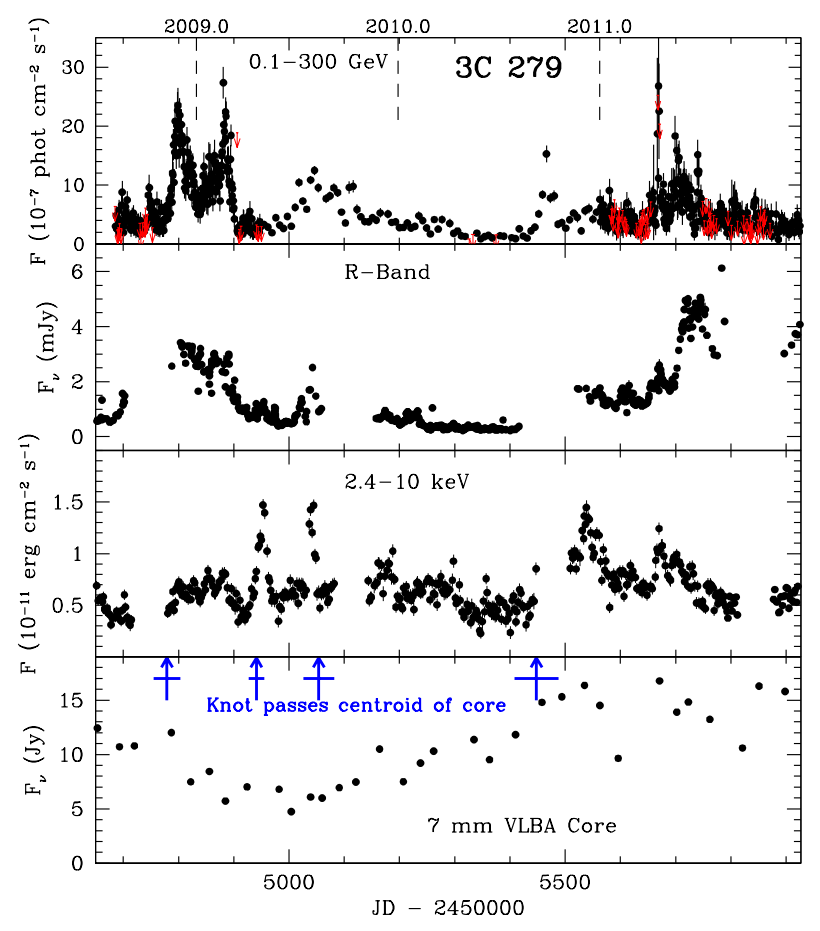

Figure 1. Multi-waveband light curves of 3C 279 over a twoyear period. Red arrows in the top panel correspond to upper limits to the $\gamma$-ray flux. Times of superluminal ejections are indicated by the vertical blue arrows, with the line segment crossing the arrow corresponding to the uncertainty in the ejection date. Note the complexity, with apparent correlations as well as events at one frequency that do not appear to have counterparts at other frequencies. (From data obtained by the author and collaborators [50])

losses of the electrons that produce the optical photons, we derive a magnetic field between 0.1 and $1 \mathrm{G}$. The typical dimensionless (i.e., relative to $m c^{2}$ ) energy $\gamma$ of the electrons that emit synchrotron radiation at an observed optical frequency of $10^{15} v_{\mathrm{obs}, 15} \mathrm{~Hz}$ is then

$$
\gamma \sim 2 \times 10^{4}\left[(1+z) v_{\mathrm{obs}, 15} /(\delta B)\right]^{1 / 2} .
$$

When a "seed" photon of frequency $10^{15} v_{\text {seed,15 }}^{\prime} \mathrm{Hz}$ as measured in the rest frame of the plasma moving relativistically down the jet is inverse Compton scattered by an electron of an energy that produces an optical synchrotron photon with observed frequency $1 \times 10^{15} \mathrm{~Hz}$, its frequency in the observer's frame is

$$
v_{\mathrm{IC}} \sim 4 \times 10^{23} v_{\text {seed }, 15}^{\prime} / B
$$

which is in the low $\mathrm{GeV}$ range. If flux variations mainly result from changes in the number of electrons in the above energy range, we then expect very similar optical and $\gamma$ ray light curves. While this is often approximately the case [e.g., 7, 11, 16, 72], the correspondence is not usually very close when examined in detail with fine time resolution.

An example illustrating this complexity is $3 \mathrm{C} 279$, whose multi-waveband light curves are displayed in Figure 1 [see also 28]. An outburst from late 2008 to early 
2009 is seen most strongly in $\gamma$-rays, not quite as strongly and with less dramatic short-term fluctuations at optical wavelengths, and weakly in X-rays. The next major event in 2009 is a sharp "orphan" (without a counterpart at other wavebands) X-ray flare. Another sharp X-ray flare a few months later corresponds to weaker optical and $\gamma$ ray flares. The first two-thirds of 2010 saw relative quiescence at all three wavebands, which ended with a sharp $\gamma$-ray flare in late 2010 . The $\gamma$-ray, X-ray, and optical light curves displayed a lot of activity over the next year, but without much correspondence among each other except for a short flare at all three spectral regions, most pronounced at $\gamma$-ray energies, in early 2011.

Chatterjee et al. [17] have studied the multi-waveband light curves of the quasar PKS 0208-512, in which two outbursts occurred at both $\gamma$-ray and optical frequencies, while a third was only significant in the $\gamma$-ray domain. They interpreted this by proposing multiple sites of flaring emission in the jet and/or variations in the magnetic field strength in the flaring region.

\section{Combining VLBI Images with Multi-waveband Light Curves}

From the example of 3C 279, we can see that the relationships of the emission across wavebands are quite complex. This greatly weakens our ability to use light curves alone in our quest to understand the physics of emission events in blazars. Fortunately, we have more sources of information than flux vs. time: multi-epoch VLBI images in both total and linearly polarized intensity, optical polarization vs. time, and spectral energy distributions at individual epochs and as a function of time. In the opinion of the author, VLBI images are the most powerful diagnostic tool among these, since they can be used alongside light curves to determine where in the jet flares occur.

Referring to events seen in VLBA images of 3C 279 at $43 \mathrm{GHz}$, we see in Figure 1 that a new superluminal knot passed through the core (the millimeter-wave core, referred to henceforth as the mm-core; the time when the position of the centroid of a knot matched that of the mmcore is called an "ejection") either very shortly before or during each of the three outbursts in 2008-09. The period of quiescence at optical to $\gamma$-ray frequencies starting in late 2009 saw no new superluminal ejections of bright knots as the mm-core flux rose by about a factor of 3. Apparently, the jet was swinging during this time, since an extremely bright knot was ejected during a sharp $\gamma$-ray flare in late 2010 and proceeded to move at a position angle more than $90^{\circ}$ less than the previous jet direction [48]. The knot remained very bright (brighter than the core for more than a year) as its motion curled toward the old path. A few months after this feature crossed the mm-core, the flux of the mm-core dropped precipitously, coinciding with the decline of a major X-ray flare. The mm-core flux recovered to its previous high level near the time of the short flare seen at all three of the higher-frequency wavebands.

During the Fermi era, the quasar PKS 1510-089 has undergone two prolonged, multi-flare outbursts when the

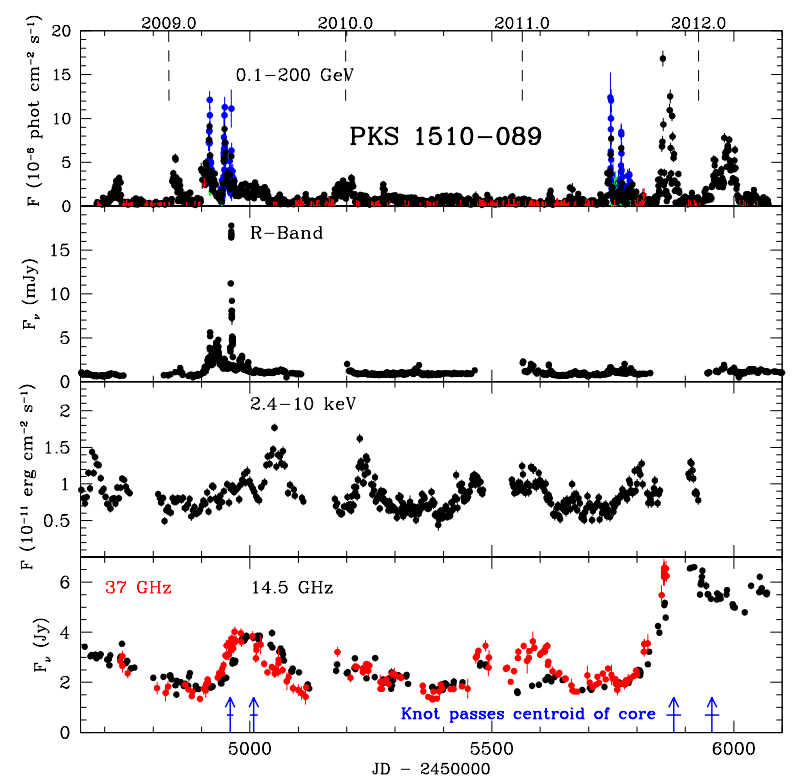

Figure 2. Multi-waveband light curves of PKS 1510-089 over a 4-year period. Times of superluminal ejections are indicated in the bottom panel by the vertical blue arrows, with the line segment crossing the arrow corresponding to the uncertainty in the ejection date. The $\gamma$-ray $(0.1-200 \mathrm{GeV})$ data from the Fermi LAT are 1-day integrations except for blue points, which are 0.25-day integrations; red arrows are upper limits. R-band optical (various telescopes) and X-ray (Rossi X-ray Timing Explorer) data are from the same sources as in [54]. In order to display the optical variations with better clarity, the extremely high-amplitude flare at JD-2450000 $=4962$, which reached $18 \mathrm{mJy}$, is truncated. [From data published in [54] and [43], as well as data not yet published from Metsähovi Radio Observatory $(37 \mathrm{GHz})$ and University of Michigan Radio Astronomy Observatory (14.5 $\mathrm{GHz})]$

$\gamma$-ray flux sometimes exceeded $1 \times 10^{-5}$ phot $\mathrm{cm}^{-2} \mathrm{~s}^{-1}$. As seen in Figure 2, the 2009 and 2011-12 outbursts each started with an optical $/ \gamma$-ray flare that occurred prior to major increases in the 14.5 and $37 \mathrm{GHz}$ radio flux. Another set of optical $/ \gamma$-ray flares coincided with a radio outburst as a new, very bright superluminal knot passed through the mm-core on $43 \mathrm{GHz}$ VLBA images. The suppression of radio emission early in the development of the outburst and the high amplitude of the $\gamma$-ray vs. optical flares at the beginning of the 2011 outburst led Nalewajko et al. [60] to propose that the early flares during an outburst take place within the BLR, well upstream of the mm-core, while later flares occur on parsec scales. This is in concert with the millimeter-wave outburst and superluminal ejections occurring during the second set of flares if the same disturbance propagating down the jet was responsible for all of the flares during at least the first half of an outburst.

We can establish whether the optical to $\gamma$-ray outbursts generally arise from superluminal knots during or after their ejection through a statistical study of a sample of blazars. Four years of VLBA images and Fermi LAT light curves of 34 blazars compiled by the author's collabora- 
tion [57] lead to the result that, out of $62 \gamma$-ray flares, $48(77 \%)$ are simultaneous (within uncertainties) with the ejection of a new superluminal knot or a major outburst in the core at $43 \mathrm{GHz}$. This conclusion, which agrees with previously results from the VLBA and EGRET in the 1990s [34], is supported by the Metsähovi Radio Observatory group's finding that outbursts at $37 \mathrm{GHz}$ usually start before $\gamma$-ray flares ([43]; see also [40] for a similar EGRET-era result). Furthermore, both the jet and $\gamma$ ray emission were quiescent during the four years in five blazars, and $86 \%$ of all of the sources exhibited contemporaneous $\gamma$-ray and mm-wave quiescent periods. In addition, Lister et al. [46] and Arshakian et al. [9] have found evidence for a general relationship between radio and $\gamma$ ray properties.

From the $43 \mathrm{GHz}$ study, we conclude that, even accounting for chance coincidences, more than $50 \%$ of $\gamma$ ray flares occur in the mm-core, which lies parsecs from the black hole in luminous blazars. This means that millimeter-wave VLBI images most of the $\gamma$-ray emitting regions. Since optical and $\gamma$-ray flares are often simultaneous, the VLBI images also image sites of variable optical synchrotron emission. A number of theorists have resisted this observational result on the grounds that intraday variability on parsec scales is difficult to understand (e.g., Tavecchio et al. [68]). However, the observation of flaring emission of photons with near-TeV energies from some quasars (e.g., 1222+216 [8] and PKS1424-418 [69]) has provided convincing evidence that such events need to take place well outside the BLR. Otherwise, the very high-energy photons would be lost to pair production off the BLR photons before they could escape to be detected at Earth.

It does appear, though, that BL Lac objects with high-frequency synchrotron SED peaks and luminous TeV emission are exceptions (see papers in these proceedings by Richards, Lico, and Niinuma). The parsec-scale jets of Mkn421 and other similar objects do not tend to produce highly superluminal knots. This does not necessarily mean that the jet flows are not highly relativistic, since many BL Lac objects (e.g., BL Lacertae itself) contain multiple stationary emission features in addition to superluminal knots. It may be the case that the plasma flow in the nearly quiescent jets is smooth for some reason.

Outbursts in the core without ejections may be the same type of disturbance that creates the appearance of superluminal knots. Perhaps the absence of a knot is caused by strong radiative energy losses that suppress the emission. The latter could occur for the strongest $\gamma$-ray flares, which should correspond to the highest energy losses from inverse Compton emission. However, as is discussed further below, there are examples of high-amplitude outbursts in the mm-core that produce neither a superluminal knot nor a counterpart at optical and $\gamma$-ray frequencies.

A blazar with clear multi-waveband connections is the quasar 3C 454.3. Figure 3 presents light curves demonstrating that the major outbursts have counterparts from millimeter to $\gamma$-ray wavelengths. Of particular interest is the very sharp "megaflare" in late 2010. The sharpness

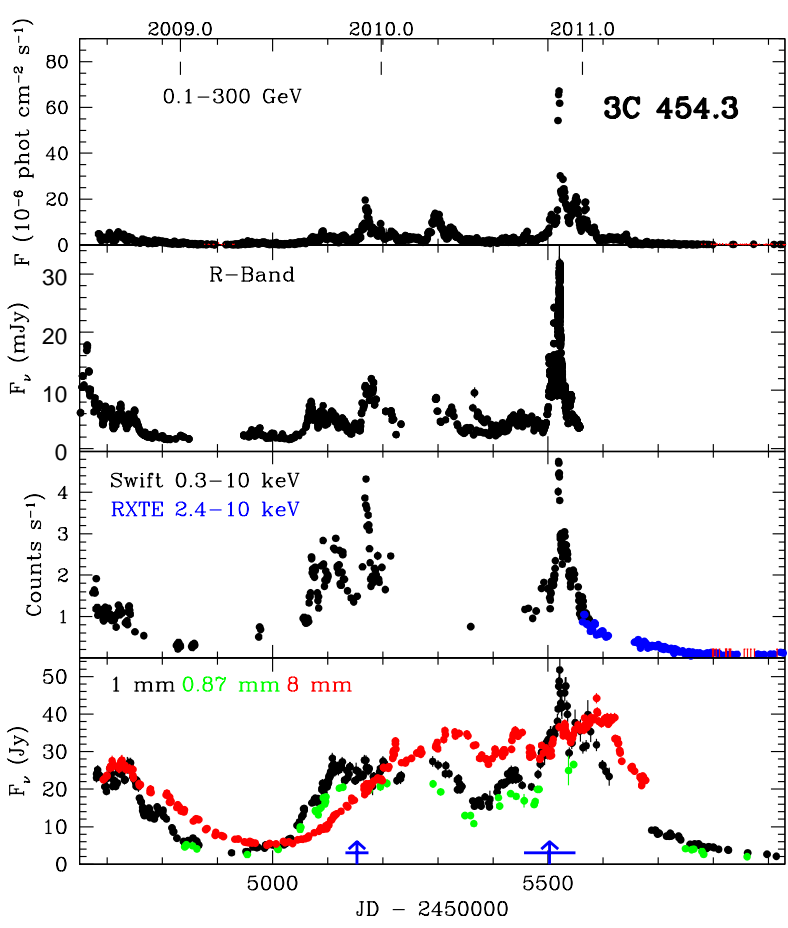

Figure 3. Multi-waveband light curves of $3 \mathrm{C} 454.3$ over a 3.5year period. Times of superluminal ejections are indicated by the vertical blue arrows; the line segment crossing the arrow corresponds to the uncertainty in the ejection date. Note that the major outbursts are apparent at all wavebands. (From data published in $[39,72])$

of the peak at $\lambda 1.3 \mathrm{~mm}$ and its simultaneity with the optical, X-ray, and $\gamma$-ray peak requires two conditions. One is that the flare must be on parsec scales rather than inside the broad emission-line region (BLR). If, as concluded by Vercellone et al. [70] based on rapid variability and SED fitting, the flare took place in a region of radius $3.6 \times 10^{16}$ $\mathrm{cm}$ located $\sim 0.05 \mathrm{pc}$ from the black hole, the optical depth at $\lambda 1.3 \mathrm{~mm}$ would have been 5600 and the event would have been impossible to detect at that wavelength [72]. The rapid decline at $\lambda 1.3 \mathrm{~mm}$ can be explained if the radiative energy losses were so severe that $\sim 50 \mathrm{MeV}$ electrons emitting at this wavelength decayed in energy within about 2 days in our rest frame. This, plus opacity effects, is probably why there was no sharp peak in the $\lambda 8 \mathrm{~mm}$ light curve and the superluminal knot ejected was not as bright as the knot ejected during the 2009 outburst [39].

The mm-core did, on the other hand, become very bright during the outburst, starting about two weeks before the sharp flare [39]. Analysis by the same authors of the time profile of the light curve of 3C 454.3 during outbursts finds a triple-flare structure, similar to that found in earlier events in the same object [37]. Super-resolved images (i.e., convolved with a point-spread function corresponding to the angular resolution of the longest baselines of the VLBA rather than with a beam resulting from formal statistical weighting) contain a triple structure in the $\mathrm{mm}$-core region that may be related to this repeating light curve pattern [37]. 


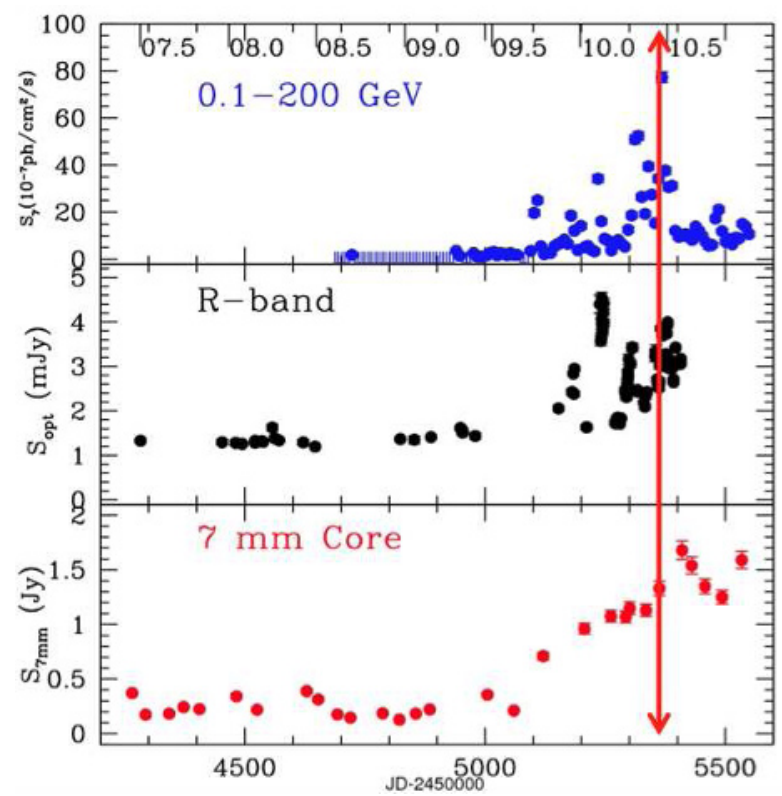

Figure 4. Multi-waveband light curves of $1222+216$ over a 4year period. The red arrow indicates the time of detection at sub$\mathrm{TeV}$ photon energies [8]. (Figure from a presentation by Jorstad et al. [38])

It is instructive to examine the relationship between high-energy flares and events in the jet at millimeter wavelengths by studying less active blazars than 3C 279 , PKS 1510-089, and 3C 454.3. One prime specimen is $1222+216$ (4C 21.35). Its jet was inactive from the time when Fermi began operating in August 2008 until September 2009 . Then the flux at $37 \mathrm{GHz}$ rather abruptly increased by more than $60 \%$ and the first of many $\gamma$-ray flares was observed. The mm-core in $43 \mathrm{GHz}$ VLBA images brightened from 0.30 to $0.75 \mathrm{Jy}$ between $2009 \mathrm{Au}-$ gust 16 and October 16. (Unfortunately, there were no VLBA observations in September.) Figure 4 presents the $\gamma$-ray, optical, and mm-core light curves. Interestingly, no new superluminal knot was detected with an ejection time near this event. Rather, a strong knot was ejected later, at 2010.4, essentially coincident with the brightest $0.1-200 \mathrm{GeV} \gamma$-ray flux and the detection of the blazar with $M A G I C$ at photon energies up to at least $0.4 \mathrm{TeV}$ [8]. While it is tempting to ascribe the absence of a bright knot associated with the late-2009 activity to radiative losses, it is then difficult to understand why the more luminous event in mid-2010 would not have suppressed the emission from the bright knot ejected at that time. Therefore, another explanation is needed.

Another blazar that woke up from a multi-waveband slumber is $0836+710$, which is featured in a paper by Jorstad et al. in these proceedings. As in $1222+216$, the $\mathrm{mm}$-core of this quasar increased in brightness along with the optical and $\gamma$-ray emission after a long quiescent period. The ejection of a very bright superluminal knot occurred near the time of the first flare of the flux outburst. About six months later, the polarization electric-vector position angle (EVPA) in the knot rotated along with the
EVPA of the variable optical emission. This implicates the knot as the site of the high-frequency flares, even when the knot was $\sim 35 \mathrm{pc}$ (after deprojection) from the mm-core.

In 0836+710 and some other blazars (e.g., 1156+295 and CTA102), some major outbursts in the mm-core do not coincide with outbursts at higher frequencies, while others do in the same blazar. This inconsistent behavior is difficult to understand. One possibility is that particle acceleration is too inefficient during the first type of event to generate electrons with energies high enough to radiate at optical and $\gamma$-ray frequencies. That is, energies of electrons in the range of hundreds of Mev are attained, but energies exceeding $\sim 5,000$ are not achieved. Perhaps there is a deficit of moderate-energy "seed" electrons in the $\sim 10 \mathrm{MeV}$ range that facilitate rapid acceleration to higher energies by shocks. One can imagine that the level of turbulence - and therefore second-order Fermi acceleration in the ambient jet - might vary with time upstream of the core. Alternatively, some outbursts might involve propagating shocks when the jet's bulk Lorentz factor increases after a period of slower flow, while in others the jet flow becomes denser without becoming fast enough to create a moving shock. Both of these ideas presuppose that either turbulence or a moving shock is needed to raise the energies of the seed electrons to $\sim 10 \mathrm{MeV}$ values.

\section{Linear Polarization as a Diagnostic Tool}

At radio through optical wavelengths, measurements of linear polarization ${ }^{1}$ provide a wealth of information that complements what we learn from light curves and total intensity imaging. The case of $0836+710$, discussed above, illustrates how polarization vs. time can aid in the identification on VLBI images of a feature responsible for a high-energy flare. Such cross-identification can be considered firm if either a new emission feature with a unique EVPA appears in VLBI images and if the EVPA is the same as found at optical wavelengths at the same time, or if the polarization vector of the feature rotates in the same manner as at optical wavelengths. This led the author and collaborators [53] to identify optical, X-ray, and possibly sub-TeV $\gamma$-ray flares in BL Lacertae with a new superluminal knot while it was upstream of the core on $43 \mathrm{GHz}$ VLBA images.

It is common for the optical EVPA to be similar to that seen in the mm-core or, less frequently, in a superluminal knot $[20,36]$. Often, the direction of the polarization vector lies either nearly parallel or perpendicular to the local jet axis. In the mm-core, this can be understood if the core corresponds to a standing conical shock (often called a "recollimation" shock, but can also be a decollimation shock or one type of shock followed by another [14]) [12, 13, 63]. Cawthorne et al. [14] have taken one step further by comparing polarized intensity images of

${ }^{1}$ This review does not cover circular polarization, which is observed at radio wavelengths, since a study of a large sample of blazars has found that circular polarization appears to be unrelated to the multi-waveband properties of jets [29]. The use of circular polarization as a diagnostic of the jet magnetic field is discussed by Homan et al. [30] and in the review paper by Wardle in these conference proceedings. 
the BL Lac object $1803+784$ observed by the VLBA with simulated images based on such a model, with favorable results. The implication of these studies is that much of the optical emission in blazars occurs in the mm-core. This conclusion is quite surprising given the placement of the $\mathrm{mm}$-core parsecs from the central engine, which is hard to reconcile with the rapid variability of blazars in both flux and polarization at optical wavelengths.

The tendency of the linear polarization E-vectors to line up with the jet axis in BL Lac objects and to lie roughly transverse to the jet axis in quasars (e.g., [23]) is not as strong at $43 \mathrm{GHz}$ as at lower frequencies [58]. This suggests that the effect is stronger well downstream of the mm-core. The difference could be the magnetic field geometry in the ambient jet through which shocks propagate, with quasar jets supporting more longitudinal fields (perhaps from velocity shear across the jet) and BL Lac objects more subject to turbulence generating chaotic fields. Gabuzda (in the proceedings of this conference) promotes the idea, with supporting evidence from Faraday rotation and circular polarization, that the field maintains a helical structure, which can yield polarizations either parallel or perpendicular to the jet axis under different circumstances. However, support for either scenario is ambiguous without a robust explanation for the dichotomy beween $\mathrm{BL}$ Lac objects and quasars. Strong evidence for a toroidal (= helical with a high pitch angle) magnetic field geometry comes from rotations in the polarization vector observed in a number of blazars. While apparent rotations can occur owing to random fluctuations in magnetic field direction, e.g., those expected from turbulence [32], repeated smooth rotations - especially in the same direction and with non-random timing (e.g., simultaneous with a flare) - implicates a physical mechanism as the cause. In the case of BL Lacertae, the optical polarization vector rotated by $\sim 240^{\circ}$ shortly before a superluminal knot crossed the mm-core [53] during a flare. A similar, more dramatic event in PKS 1510-089, displayed in Figure 5, occurred in 2009 when the EVPA rotated through four full $180^{\circ}$ cycles during a multi-flare outburst, with the final high-amplitude flare coinciding with a bright knot passing through the mm-core.

Polarization rotation events such as these were actually predicted by Vlahakis [71] based on MHD models of jet formation. The geometry is sketched in Figure 6. An emission feature (e.g., a magnetosonic shock) that does not fill the entire cross-section of the jet (an important criterion, otherwise the polarization would cancel because of symmetry) traces out a spiral trajectory as it circulates around the helical field lines, which are advecting downstream at near-light speed. In a frame flowing with the field lines, the emission feature's path follows a loop around the nearly toroidal field. When one takes into account aberration and light-travel delays, the EVPA follows an undulating curve, shown in the bottom panel of Figure 5 for PKS 1510-089.

These observations provide the strongest observational evidence to date that the MHD jet launching scenario actually occurs in blazars. Other EVPA rotations include, among others, those observed in 3C 279 ([2, 41] and

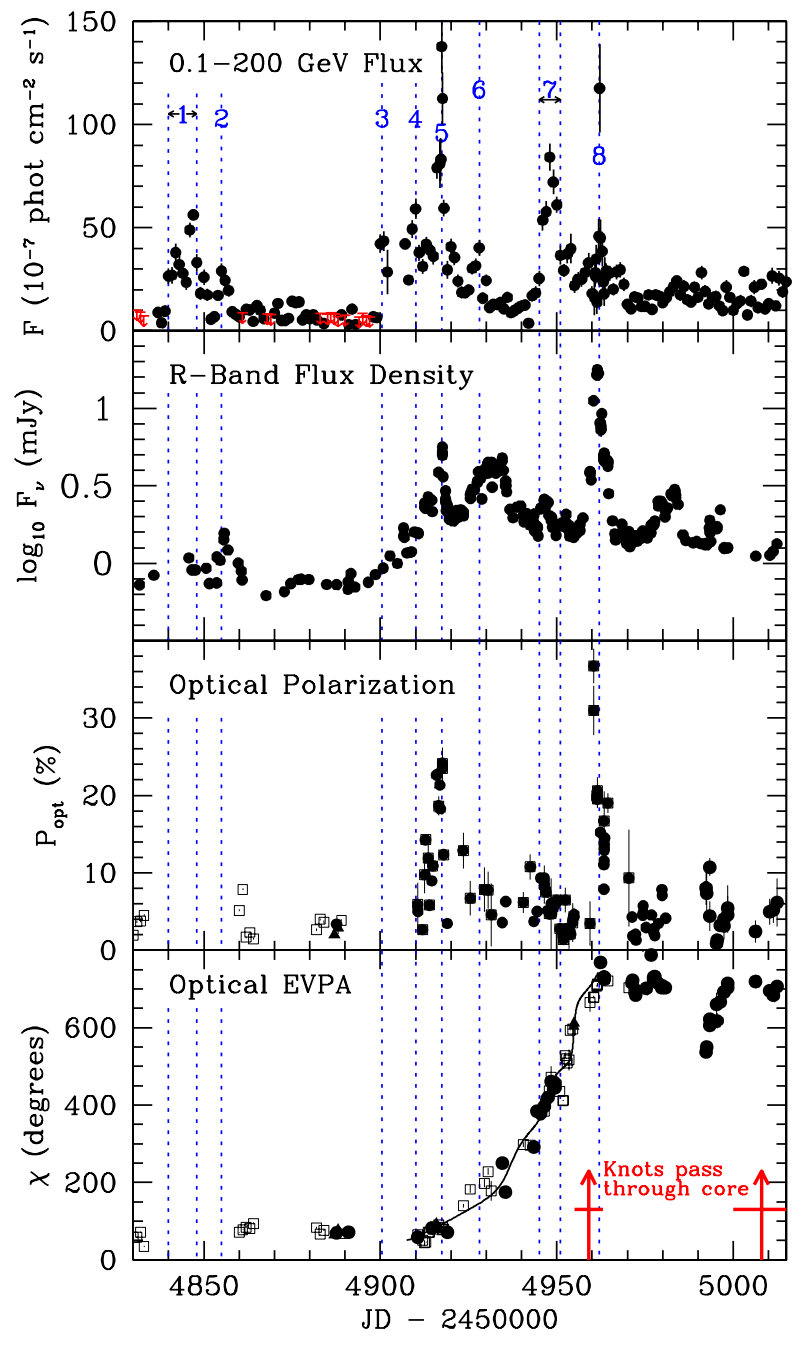

Figure 5. $\gamma$-ray and optical light curves and optical polarization vs. time of PKS 1510-089 during and after the 2009 outburst. The rotation of the linear polarization vector is seen in the bottom panel. From data presented in [54]

Kiehlmann et al., these proceedings), 0716+714 [42], and 3C 454.3 [39]. The rotations could also be caused by physical twisting of the jet, as proposed by Abdo et al. [2] and Raiteri et al. [64], the latter of which also explain flux variations in 3C 454.3 and other blazars.

Farther downstream, however, turbulence appears to rather strong in blazar jets. This is most easily seen in the mean level of linear polarization and time variations of both the degree and position angle. Consider a simplified model of turbulence that creates $N$ emitting cells in the plasma, with each cell containing a magnetic field that is uniform but whose direction is random. Although the level of synchrotron radiation depends on the angle (after relativistic aberration is taken into account) between the magnetic field and the line of sight, the polarization vector depends only on the azimuthal angle. The mean polarization $\langle P\rangle$ is then $\sim N^{-1 / 2}$ times the maximum value $P_{\max } \sim 75 \%$ [33]. If the cells are replaced by new turbulent cells, again 

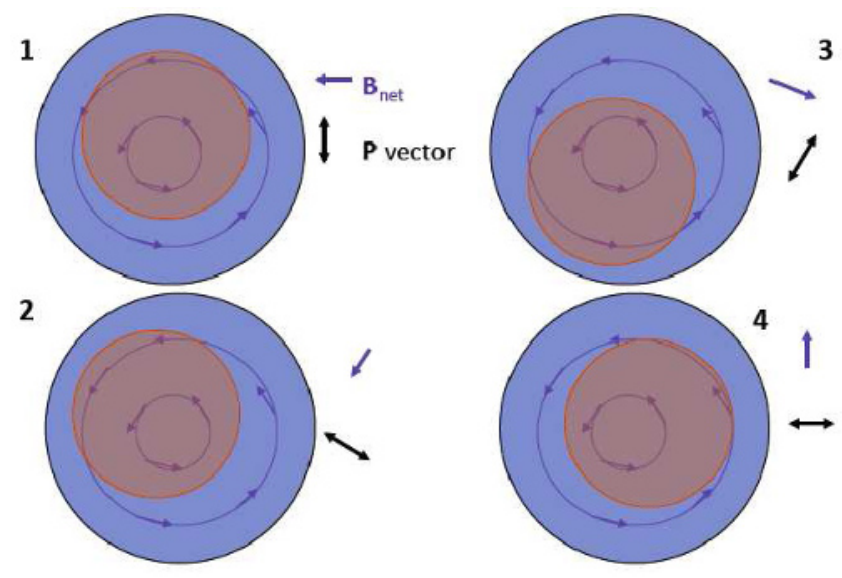

Figure 6. Sketch showing how the linear polarization vector $\mathbf{P}$ can rotate as a shock or other bright feature follows a spiral streamline as it moves down the jet. Four different times are illustrated, representing continous rotation of the feature as it moves down the jet. If the shock does not cover the entire cross-section of the jet, then the mean magnetic field vector $\mathbf{B}$ rotates, hence so does $\mathbf{P}$.

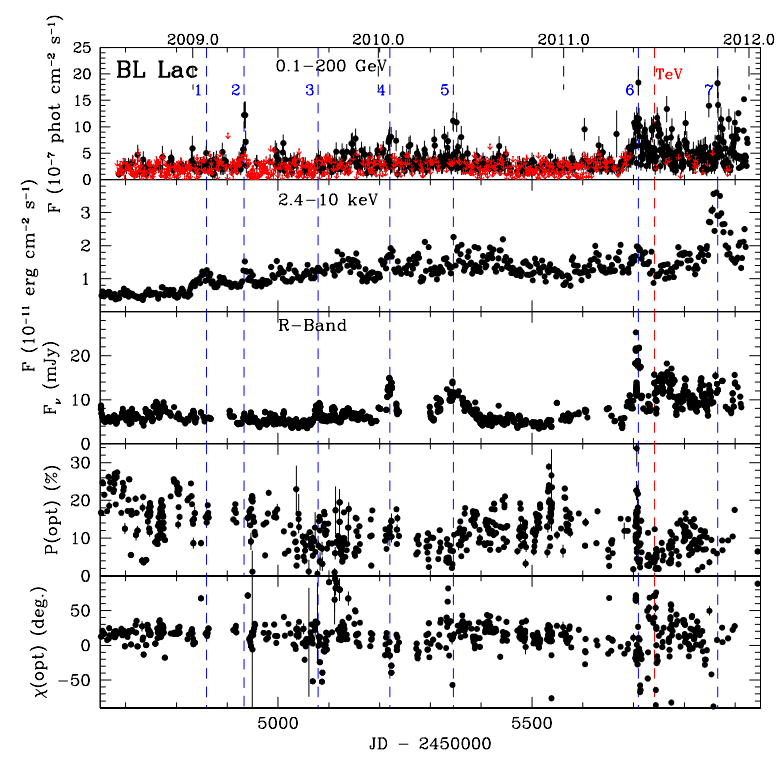

Figure 7. Multi-waveband light curves of BL Lac, including degree and position angle of optical linear polarization [51].

with randomly oriented fields on a regular basis, the degree of polarization will vary with a standard deviation equal to the mean divided by $\sim 2^{1 / 2}$, or $\langle P\rangle / 2[21,33]$. This general description agrees qualitatively with the optical polarization of blazars like BL Lacertae (displayed in Fig. 7).

\section{Spectral Energy Distributions}

After compiling a large database of multi-waveband observations, it is possible to construct SEDs of a blazar at different epochs. We invariably find the double-humped profile, as in Figure 8, with the luminosity represented by $v L_{v}$ peaking at IR and sub-GeV $\gamma$-ray frequencies in quasars and low-synchrotron-peak BL Lac objects, and at $\mathrm{X}$-ray and sub-TeV energies in high-synchrotron-peak BL Lac objects. This fits in with the standard emission model of synchrotron radiation at lower frequencies and inverse Compton scattering at high energies. During outbursts, the $\gamma$-ray luminosity dominates by up to 3 orders of magnitude over the synchrotron component in quasars, while for most BL Lac objects the $\gamma$-ray and synchrotron luminosities are more comparable [1].

If the main $\gamma$-ray emission mechanism is synchrotron self-Compton scattering (SSC), i.e., inverse Compton scattering of seed photons produced via synchrotron radiation in the same location as where they are scattered, high levels of $\gamma$-ray dominance would require so many electrons that there would be high optical depths to both scattering and pair production [27]. The latter would produce more radiation and more pairs, leading to a "fireball" cascade, and most of the luminosity would emerge at low MeV and soft X-ray energies. This has led to the conclusion that the seed photons in quasars come from slowly moving sources located outside the site of the scattering: the accretion disk [22], the BLR [65], a dusty molecular torus [10], a relative slow sheath of the jet [24], or a Mach disk (a strong shock oriented perpendicular to the flow, located at the axis and covering only a small fraction of the jet cross-section [19]) $[51,52]$. A great advantage of this "external" Compton scattering (EC) is that the seed photons are blueshifted in the frame of the jet plasma, as long as the site of scattering is not much farther down the jet than the distance of the seed photon source from the central engine. This greatly increases the $\gamma$-ray luminosity. However, the constraint on the relative location is a problem for the accretion disk and BLR origins of the seed photons if the $\gamma$-ray flares occur on parsec scales. Even IR photons from the dusty torus could strike the jet at too wide an angle to produce the high $\gamma$ ray luminosities observed during outbursts if the torus is confined to a region less then 2-3 parsecs from the central engine, as may be the case in the quasar 1222+216 [49].

For high-synchrotron-peak BL Lac objects, however, SSC models often produce reasonable fits to the SEDs, and $\mathrm{X}$-ray variations are generally well-correlated with those in the TeV range (e.g., [3, 4]). The $\gamma$-rays (and perhaps $\mathrm{X}$-rays) observed in low-synchrotron-peak BL Lac objects could also be of SSC origin, since the ratio of $\gamma$-ray to synchrotron luminosities is usually of order unity [6, 7], although Ackermann et al. [5] have proposed EC with seed photons from a dusty torus for $\gamma$-rays and Compton scattering of accretion disk photons by low-energy pairs for $\mathrm{X}$-rays in $\mathrm{AO} 0235+164$.

It has become customary for observational teams to enlist a theorist to fit the SED of a blazar at one or more epochs to justify a particular interpretation of the emission process and location. The author's feeling is that, while this exercise is sometimes important, it can be falsely reassuring. Furthermore, it takes up a considerable amount of the theorist's time that could be more profitably spent devising new models that can explain more than just the 


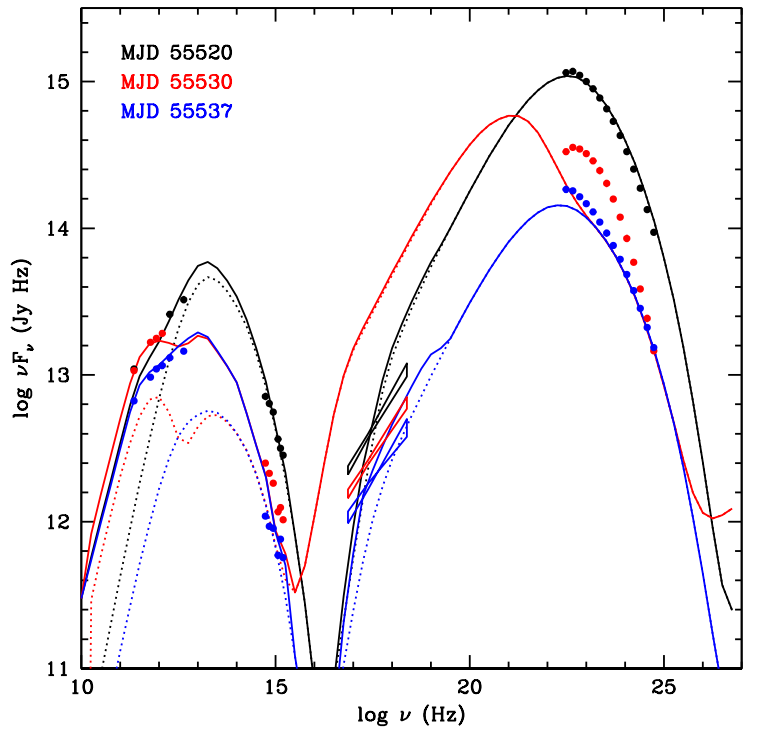

Figure 8. Example illustrating how of model fits to an SED can be deceptive. The model reproduces the SED fairly well (except for the X-ray slope) at two epochs (black and blue symbols and lines), but the fit to the third epoch data is terrible at X-ray and $\gamma$-ray frequencies. (The dotted curves represent the rapidly variable emission in the model, while the solid curves add much more slowly varying components that are simply added to the model.) The filled circles correspond to observations of $3 \mathrm{C} 454.3$ presented by Wehrle et al. [72].

SED. This is because the ability to fit a model SED to some observations (the word "fit"here is approximate and subjective, since all, or nearly all, published SED fits would fail a $\chi^{2}$ test) does not mean that the physical conditions of the blazar were similar to the model parameters. The fitting of the SED of the mega-outburst of 3C 454.3 in late 2010 discussed in the previous section is a prime example: good SED fit, bad conclusion about the size and location of the emission region responsible for the flare. We should demand that successful models explain not only the SEDs, but also the time variability of flux, polarization, and jet structure. And we should give theorists time to develop models that show promise in doing so.

Nevertheless, the inability to fit an SED despite a parameter search, or to fit the SED at one epoch but not another, is a sign that a model might be inadequate. An example is an attempt by the author to fit the SED of 3C 454.3 at three epochs during the 2010 outburst, illustrated in Figure 8. The main source of seed photons in the model is synchrotron plus SSC emission from the Mach disk. The excess hard X-ray flux and overly flat X-ray spectrum of the model are worrisome, but perhaps this could be fixed by altering the energy distribution of electrons in the 100 s of $\mathrm{MeV}$ range. The more serious problem is the shifting of the inverse Compton spectrum up and to the left some of the time, an effect that was not observed. This flaw would not have been recognized if the numerical code used to calculate the SED were applied only to specific times when an observed SED was available, with parameter values adjusted separately for each epoch.

The preference of many theorists for a thermal source of seed photons arises partly from SED fitting. The inadequate fits at X-ray energies of the model shown in Figure 8 is largely driven by the synchrotron spectrum of the seed photons, which is much broader-band than a thermal spectrum. Scattering of the plentiful photons at frequencies between $10^{11}$ and $10^{12} \mathrm{~Hz}$ generates a strong X-ray flux. The observed high-energy hump, on the other hand, peaks at an energy of $\sim 100 \mathrm{MeV}$ and drops by $\sim 2$ orders of magnitude into the X-ray domain during an outburst. This is easiest to model if the seed photons have a thermal distribution.

The origin of the X-ray emission from blazars that do not belong to the high-synchrotron-peak class has still not been decided. The spectral (energy) indices tend to lie in the range of $0.7 \pm 0.02$, which is similar to the millimeter-submillimeter spectral indices of $\gamma$-ray bright blazars [44]. This is what one expects from SSC models, where millimeter-wave to IR photons are scattered to X-ray energies by electrons in the $100 \mathrm{~s}$ of $\mathrm{MeV}$ range. However, the lack of consistent correlations between millimeter-wave and X-ray variations in the light curves presented in Figures 1-3 implies that multiple processes may be reponsible for generating X-rays at different times in a given blazar. Unfortunately, with the demise of RXTE there are no space missions dedicated to continuous $\mathrm{X}$-ray monitoring now that ALMA is available to monitor at $\mathrm{mm} / \mathrm{submm}$ wavelengths.

The millimeter-wave to optical SEDs of 3C 454.3 displayed in Figure 8 can be fit by piece-wise power-laws, but the break(s) required by the steepness of the opticalIR vs. mm-wave spectra is considerably greater than the value of 0.5 expected from radiative energy losses [55]. This issue has received little attention, but imposes an important constraint on particle acceleration scenarios. The author's own solution has been to propose that, in addition to radiative losses steepening the spectrum somewhat, the volume filling factor decreases toward higher electron energies/higher frequencies in the particle acceleration region.

\section{The Case for Turbulence as a Major Factor in Blazar Jets}

As discussed above, time variations of the optical polarization of blazars provides considerable evidence for a chaotic component to the magnetic field, as would be expected from turbulence. Furthermore, power spectra of blazar variations can be fit by power laws $[15,16]$, a fact that suggests that the flux changes are governed by a noise process rather than occasional discrete highamplitude events.

The author has used this evidence as a basis for developing a new paradigm for blazar variability $[50-52,56]$. The power spectrum of flux variations is assumed to reflect time variations of the energy density of relativistic electrons injected into the jet. An approximate treatment 
of turbulence divides the plasma into many cells, each of which has a uniform magnetic field whose direction is selected randomly every ten time steps and rotated smoothly in between. The plasma is advected at the systemic bulk velocity of the jet flow across a standing conical shock. (For determining the Doppler factor, each cell is given a randomly directed turbulent velocity that is added to the systemic velocity.) At the shock front, electrons are assumed to be accelerated with a power-law energy distribution up to a maximum energy that depends on the angle between the magnetic field and the shock normal. (Alternatively, the maximum energy can be determined randomly within a given probability distribution.) This dependence on field direction approximates the results from particle acceleration studies (e.g., [66, 67]) that particle acceleration is more efficient when the field is nearly parallel to the shock normal (as measured in the rest frame of the shock). As the plasma advects downstream of the shock, the electrons lose energy via synchrotron and inverse Compton radiation. Seed photons from a torus containing hot dust and from nonthermal emission in a Mach disk are included in the inverse Compton radiation. A numerical code, TEMZ (Turbulent Extreme Multi-Zone model), calculates the time-dependent emission from microwave to $\gamma$-ray frequencies, as well as the linear polarization at microwave to optical wavelengths, as a function of time.

A sample plot of the simulated flux and polarization vs. time evolution of a blazar computed by the TEMZ code is displayed in Figure 9. Note in the figure that there are both correlated variations and deviations in the fluctuations at different wavebands, a characteristic of blazars noted in $\S 2$. In addition, the polarization position angle executes apparent rotations in both directions. The results look encouragingly similar to observations of BL Lac (Fig. 7), so the author plans to explore parameter space to determine the extent to which the model can explain the range of behavior seen in actual blazars.

Trial SEDs of a variable component of a blazar, obtained with the TEMZ model, are denoted by the dotted lines in Figure 8. One can see that the slope of the mmwave to optical SED changes by much more than 0.5 despite the injection of a simple power-law energy distribution. This is due to the lower volume filling factor of higher-energy electrons caused by the dependence of the maximum energy on magnetic field direction relative to the shock normal. This, as well as the stronger effect that radiative losses have on higher energy electrons, also has an effect on the polarization, since fewer cells are involved in the emission at higher frequencies. The effect causes a higher mean degree of polarization and also stronger variations in the degree and position angle, as well as the flux, at higher frequencies, as observed [39, 56].

Narayan \& Piran [62] have suggested that a model similar to TEMZ can explain the most extreme intraday variability of blazars on parsec scales. In order to enhance the beaming, they propose that the turbulence is supersonic, so that the emission from some of the turbulent cells is especially well beamed. An alternative that can serve the same
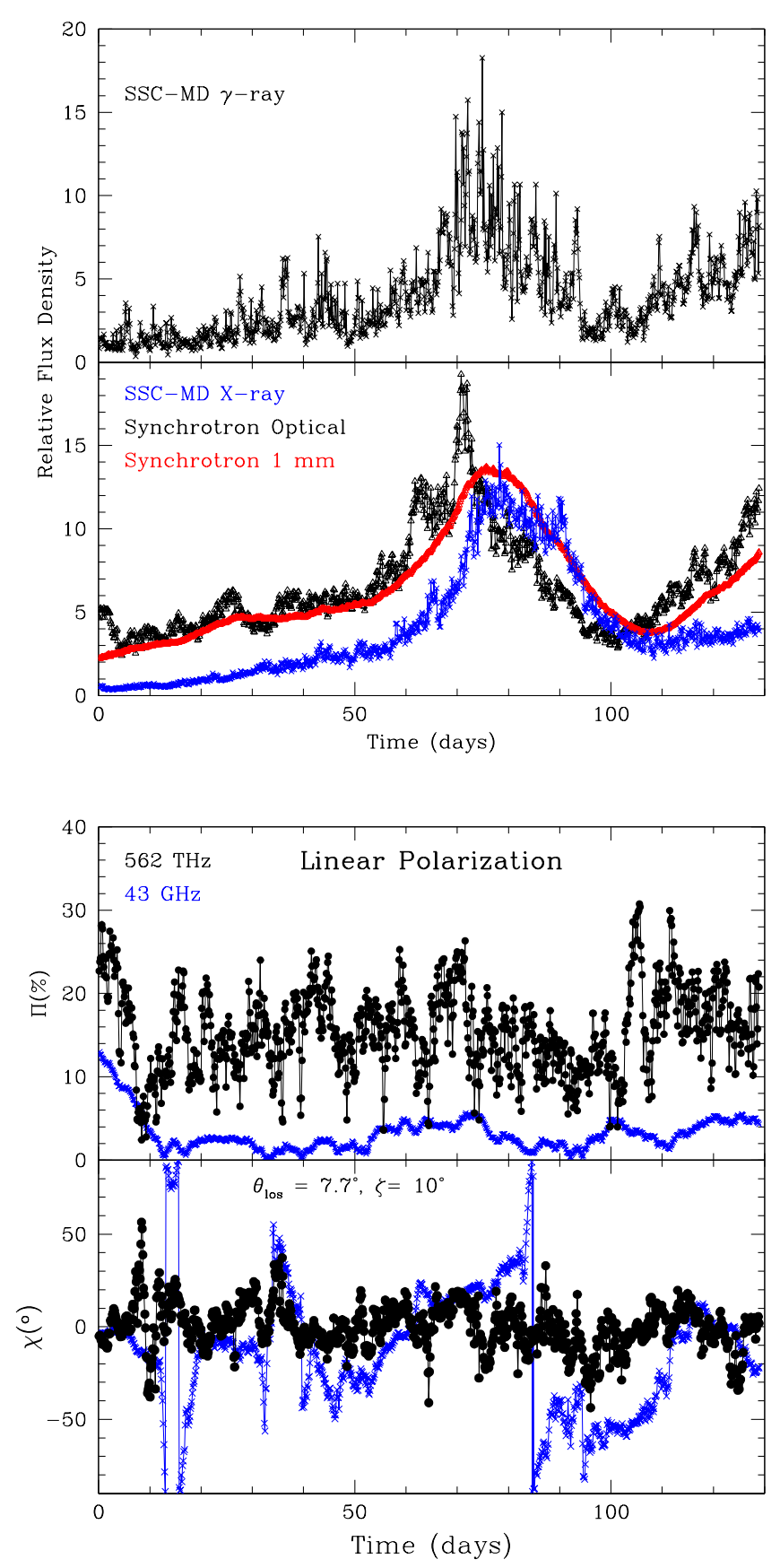

Figure 9. Sample simulated flux and polarization vs. time curves from the author's TEMZ model. For reference, $562 \mathrm{THz}$ corresponds roughly to the optical $\mathrm{V}$ band.

purpose is magnetic reconnection, which may be more efficient at accelerating electrons to very high energies than are relativistic shocks [26]. The author hopes that simulations of reconnection events will soon become sophisticated enough to make predictions regarding the polarization characteristics so that the model can be compared with the data, as is now possible with the turbulence scenario.

Another factor to consider regarding variability on parsec scales is that the jets of blazars are extremely narrow, with opening half-angles $\sim 0.1 / \Gamma_{\text {flow }}[18,35]$. This indi- 
cates that the jet width $1 \mathrm{pc}$ from the black hole is only $\sim 6 \times 10^{16}\left(\Gamma_{\text {flow }} / 10\right)^{-1} \mathrm{~cm}$. A mean optical polarization of $\sim 10 \%$, typical of very active blazars, implies $N \sim 50$ cells with randomly oriented magnetic fields, so each cell has a diameter of about $1 / 7$ of the jet width, or a bit less than $10^{16} \mathrm{~cm}$, or $\sim 3$ light-days. The time-scale of variability of the cell can then be as short as $\sim 4(1+z) /(\delta / 20)$ hours. So, intraday variations are possible in such a model. Of course, if only $\sim 2 \%$ of the cells are varying, the amplitude of the variability is not high. But if the varying cell has a turbulent velocity component in our direction, or if random flucuations cause a number of cells to vary in the same way simultaneously in our frame (i.e., when lighttravel delays are taken into account) at some epoch, highamplitude intraday variability can happen on an irregular basis.

A likely source of turbulence is current-driven instabilities at end of the zone where the jet is accelerated and collimated [59, 61]. By this location, the magnetic energy density has dropped until it is comparable to the kinetic energy density of the plasma. Turbulence tends to drive a plasma toward equipartition between the magnetic and internal particle energy densities. Magnetic reconnections are also likely to occur in this region, since as the field becomes more chaotic there is a greater chance for sections of the plasma with oppositely directed fields to come in contact with each other. The mm-core of the jet could simply be the location where turbulence and reconnections efficiently accelerate electrons, but the agreement of the observed linear polarization with that calculated for a turbulent flow crossing a standing conical shock [14] suggests the presence of such a shock in the mm-core.

\section{Conclusions}

As a community, we blazar enthusiasts are now accumulating an extremely rich dataset that should provide enough clues for us to figure out both the general and detailed physics of relativistic jets in blazars. In order to accomplish this ultimate goal, theoretical models need to catch up to observations. But so far, attempts to do this have instead added to the paradoxes that have haunted us since highly variable quasars were first identified as cosmologically distant objects.

The observations now indicate that most (but probably not all) outbursts and flares occur on parsec scales. What is the source of seed photons at these distances from the central engine? Thermal emission from hot dust extends to parsec scales, but if it is confined to a torus, it is not sufficiently blueshifted in the frame of plasma more than $\sim 1 \mathrm{pc}$ from the black hole to produce the observed $\gamma$-ray luminosities during outbursts. Relatively slow sections of the jet, e.g., a Mach disk, might provide ample seed photons, but the SEDs are better fit by a thermal spectrum of seed photons. One possible solution, supported by reported variations in emission lines from 3C 454.3 during its late-2010 mega-outburst [31, 45], is that stray broad emission-line clouds are located near the parsec-scale jet and are illuminated by beamed UV photons from the jet.
This could provide a variable source of seed photons (as the Mach disk does in the TEMZ model), thus affecting the $\gamma$-ray variations. This is similar to a model by Ghisellini \& Madau [25], except that more than one cloud might be involved and the seed photon variations can come from previous flares rather than the current one since light-travel delays are involved.

It would be very satisfying to write that our recent advances allow us to answer all of the questions posed in the introduction. But this is only honestly possible for some, and of course further counter-evidence could be uncovered in the near future to cast these in doubt.

1. Is there strong evidence for most theorists' belief that jets are launched, accelerated, and collimated by the twisting of magnetic field lines owing to the differential rotation of plasma near the black hole? Yes - the rotations of optical polarization vectors, e.g., in BL Lac and PKS 1510-089 prior to a knot reaching the $\mathrm{mm}$-core, is just what the theorist ordered!

2. How are the particles that produce the high-energy emission accelerated - at shock fronts, via turbulence, or during magnetic reconnections? There is still no general agreement on this.

3. What are the dynamics of the flow in the jet? How common are moving and stationary shocks? What role do instabilities play in the emission and structure of jets? We have accumulated a lot of clues regarding the jet dynamics, especially with the large MOJAVE survey [47], but need some time to digest the results of this and other observational studies.

4. What is the jet power? This depends crucially on the proton vs. positron content of the jet, which has not been established to the satisfaction of a majority of blazar experts.

5. Are the $\gamma$ rays from inverse Compton scattering by relativistic electrons, secondary processes initiated by protons, or even proton synchrotron radiation? The $\gamma$-ray emission makes sense if it is inverse Compton scattering by electrons, and the alternative of hadron-dominated jets boosts the power requirements by $\sim 2$ orders of magnitude. But until we identify the source of seed photons and develop models that explain the complex time variations adequately, we cannot close the door on protons causing most of the mischief.

6. Do outbursts at $\gamma$-ray and lower frequencies occur mostly on parsec scales? If so, how can the $\gamma$-ray flux be so highly variable when it arises in a region so distant from the black hole? If only a small fraction of the jet cross-section is involved, how can such a small region generate such a high apparent luminosity? The author has tried to answer this question in this review, but expects that many experts in the field doubt that the answer is correct. 
Although we have become data-rich rather suddenly, like the nouveau riche we have not yet learned how to use our treasure. And we need still more data, since variability studies often find that conclusions drawn on a data train several years long are misleading. Unfortunately, given tight funds at the US National Science Foundation and NASA, the VLBA and Fermi - two of our most valuable and unique facilities in terms of the observational parameter space that they cover - are in danger of being terminated. NASA has already shut down the great X-ray monitoring satellite RXTE, and the University of Michigan has closed its radio observatory, both of which are serious setbacks to our enterprise. Although all of the attendees at the conference, and many other astrophysicists, consider blazars to be one of the universe's great gifts to sentient beings, we need to make an even stronger case for jet studies than we have so far. And we need to lobby the astronomical community and funding agencies to keep our best time-domain instruments operating!

\section{Acknowledgments}

The author apologizes to his collaborators whose data appear in some of the figures of this paper prior to their publication in journals that will give them all proper credit for their considerable efforts. The inclusion of their data allows the review to be current, while the exclusion of the observers from the author list prevents them from being blamed for opinions expressed within the paper. The author gratefully acknowledges funding support from NASA through Fermi Guest Investigator Program grants NNX11AQ03G (observations) and NNX12AO79G (theory). The VLBA is operated by the National Radio Astronomy Observatory. The National Radio Astronomy Observatory is a facility of the National Science Foundation operated under cooperative agreement by Associated Universities, Inc.

\section{References}

[1] Abdo, A.A., et al., Astrophys. J. 463, 919 (2010)

[2] Abdo, A.A., et al., Nature 716, 30 (2010)

[3] Acciari, V.A., et al., Astrophys. J. 738, 25 (2011)

[4] Acciari, V.A., et al., Astrophys. J. 738, 169 (2011)

[5] Ackermann, M., et al., Astrophys. J. 751, 159 (2012)

[6] Agudo, I., et al., Astrophys. J. Lett. 726, L13 (2011)

[7] Agudo, I., et al., Astrophys. J. Lett. 735, L10 (2011)

[8] Aleksic, A., et al., Astrophys. J. Lett. 730, L8 (2011)

[9] Arshakian, T.G., et al., Astron. Astrophys. 537, A32 (2012)

[10] Blazejowski, M., Sikora, M., Moderski, R., \& Madejski, G.M., Astrophys. J. 545, 107 (2000)

[11] Bonning, E.W., et al., Astrophys. J. Lett. 697, L81 (2009)

[12] Cawthorne, T.V., \& Cobb, W.K. Astrophys. J. 350, 536 (1990)

[13] Cawthorne, T.V., MNRAS 367, 851 (2006)
[14] Cawthorne, T.V., Jorstad, S.G., \& Marscher, A.P., Astrophys. J. 772, 14 (2013)

[15] Chatterjee, R., et al., Astrophys. J. 689, 79 (2008)

[16] Chatterjee, R., et al., Astrophys. J. 749, 191 (2012)

[17] Chatterjee, R., Nalewajko, K., \& Myers, A.D., Astrophys. J. Lett. 771, L25 (2013)

[18] Clausen-Brown, E., et al., in Proceedings of the 11th EVN Symposium, arXiv:1301.5751 (2013)

[19] Courant, R., and Friedrichs, D.B., Supersonic Flow and Shock Waves (Springer-Verlag, New York, 1976)

[20] D'Arcangelo, F.D. "Correlated Multiwavelength Polarization in Blazars," Ph.D. Thesis, Boston U. (2010)

[21] F.D. D'Arcangelo et al., Astrophys. J. Lett. 659, L107 (2007).

[22] Dermer, C.D., Schlickeiser, R., \& Mastichiadis, A., Astron. Astrophys. 256, L27 (1992)

[23] Gabuzda, D.C., \& Cawthorne, T.V., 2000, MNRAS 319, 1056 (2000)

[24] Ghisellini, G., Tavecchio, F., \& Chiaberge, M., Astron. Astrophys. 432, 401 (2005)

[25] Ghisellini, G., \& Madau, P., MNRAS 280, 67 (1996)

[26] Giannios, D., Uzdensky, D.A., \& Begelman, M.C., MNRAS 395, L29 (2009)

[27] Guilbert, P. W., Fabian, A. C., \& Rees, M. J., MNRAS 395, 593 (1983)

[28] Hayashida, M., et al., Astrophys. J. 754, 114 (2012)

[29] Homan, D.C., \& Lister, M.L., Astron. J. 131, 1262 (2006)

[30] Homan, D.C., Lister, M.L., Aller, H.D., Aller, M.F., \& Wardle, J.F.C., Astrophys. J. 696, 328 (2009)

[31] Isler, J., et al., Astrophys. J., in press (2013)

[32] Jones, T.W., Astrophys. J. 332, 788 (1988)

[33] Jones, T.W., et al., Astrophys. J. 290, 627 (1985)

[34] Jorstad, S.G., et al., Astrophys. J. Supp. 134, 181 (2001)

[35] Jorstad, S.G., et al., Astron. J. 130, 1418 (2005)

[36] Jorstad, S.G., et al., Astron. J. 134, 799 (2007)

[37] Jorstad, S.G., et al., Astrophys. J. 715, 362 (2010)

[38] Jorstad, S.G., et al., Bull. Amer. Astron. Soc. 43, 408.04 (2011)

[39] Jorstad, S.G., et al., Astrophys. J. 773, 147 (2013)

[40] Lähteenmäki, A., \& Valtaoja, E., Astrophys. J. 590, 95 (2003)

[41] Larionov, V.M., et al., Astron. Astrophys. 492, 389 (2008)

[42] Larionov, V.M., et al., Astrophys. J. 768, 40 (2013)

[43] León-Taveres, J., et al., Astron. Astrophys. 532, A146 (2011)

[44] León-Taveres, J., et al., Astrophys. J. 754, 23 (2012)

[45] León-Taveres, J., et al., Astrophys. J. Lett. 763, L36 (2013)

[46] Lister, M.L., et al., Astrophys. J. 742, 27 (2011)

[47] Lister, M.L., et al., Astron. J., in press (2013)

[48] Lu, R.-S., et al., Astrophys. J. 772, 13 (2013)

[49] Malmrose, M.P., Marscher, A.P., Jorstad, S.G., Nikutta, R., \& Elitzur, M. Astrophys. J. 732, 116 (2011) 
[50] Marscher, A.P., in 2011 Fermi Symposium, eConference C110509, ed. A. Morselli, arXiv:1201.5402 (2012)

[51] Marscher, A.P., in 2012 Fermi Symposium, eConference C121028, ed. N. Omodel, T. Brandt, \& C. WilsonHodge, arXiv:1304.2064 (2013)

[52] Marscher, A.P., Astrophys. J., submitted (2013)

[53] Marscher, A.P., et al., Nature 452, 966 (2008)

[54] Marscher, A.P., et al., Astrophys. J. Lett. 710, L126 (2010)

[55] Marscher, A.P., \& Gear, W.K., Astrophys. J. 298, 114 (1985)

[56] A.P. Marscher and S.G. Jorstad, in Fermi Meets Jansky: AGN in Radio and Gamma Rays, ed. T. Savolainen, E. Ros, R.W. Porcas, and J.A. Zensus (Max-Planck-Institut für Radioastronomie, Bonn), p. 171, arXiv:1005.5551 (2010).

[57] A. P. Marscher, S.G. Jorstad, I. Agudo, N.R. MacDonald, \& T.L. Scott, in Proceedings of Fermi $\mathcal{E}$ Jansky: Our Evolving Understanding of AGN, ed. R. Ojha, D. Thompson, \& C.D. Dermer, eConference C1111101, arXiv:1204.6707 (2012).

[58] Marscher, A.P., Jorstad, S.G., Mattox, J.R., \& Wehrle, A.E., Astrophys. J. 577, 85 (2002)
[59] Mizuno, Y., Hardee, P.E., \& Nishikawa, K.-I., Astrophys. J. 734, 19 (2011)

[60] Nalewajko, K., et al., Astrophys. J. 760, 69 (2012)

[61] Nalewajko, K., \& Begelman, M.C., MNRAS 427, 2480 (2012)

[62] Narayan, R., \& Piran, T., MNRAS 420, 604 (2012).

[63] Polko, P., Meier, D.L., \& Markoff, S., Astrophys. J. 723, 1343 (2010)

[64] Raiteri, C., et al., Astron. Astrophys. 534, A87 (2011)

[65] Sikora, M., Begelman, M.C., \& Rees, M.J., Astrophys. J. 421, 153 (1994)

[66] Sironi, L., Spitkovsky, A., \& Arons, J., Astrophys. J. 771, 54 (2013)

[67] Summerlin, E.J., \& Baring, M.G., Astrophys. J. 745, 63 (2012)

[68] Tavecchio, F., Ghisellini, G., Bonnoli, G., \& Ghirlanda, G., MNRAS 405, L94 (2010)

[69] Tavecchio, F., et al., MNRAS 435, L24 (2013)

[70] Vercellone, S., et al., Astron. Astrophys. 736, L38 (2011)

[71] Vlahakis, N., in Blazar Variability Workshop II: Entering the GLAST Era, ed. H.R. Miller et al., Astron. Soc. Pac. Conf. Ser., 350, 169 (2006)

[72] Wehrle, A.E., et al., Astrophys. J. 758, 72 (2012) 